\title{
A double-blind, randomized, multicenter, Italian study of frovatriptan versus rizatriptan for the acute treatment of migraine
}

\author{
Lidia Savi • Stefano Omboni $\cdot$ Carlo Lisotto • \\ Giorgio Zanchin · Michel D. Ferrari • \\ Dario Zava $\cdot$ Lorenzo Pinessi
}

Received: 8 April 2010/Accepted: 7 July 2010/Published online: 5 August 2010

(C) Springer-Verlag 2010

\begin{abstract}
The objective of this study was to assess patient satisfaction with acute treatment of migraine with frovatriptan or rizatriptan by preference questionnaire. 148 subjects with a history of migraine with or without aura (IHS 2004 criteria), with at least one migraine attack per month in the preceding 6 months, were enrolled and randomized to frovatriptan $2.5 \mathrm{mg}$ or rizatriptan $10 \mathrm{mg}$ treating 1-3 attacks. The study had a multicenter, randomized, double-blind, cross-over design, with treatment periods lasting $<3$ months. At the end of the study, patients assigned preference to one of the treatments using a questionnaire with a score from 0 to 5 (primary endpoint).
\end{abstract}

Electronic supplementary material The online version of this article (doi:10.1007/s10194-010-0243-y) contains supplementary material, which is available to authorized users.

L. Savi · L. Pinessi $(\square)$

Neurology II, Headache Center, Department of Neuroscience, University of Turin, Via Cherasco, 15, 10126 Turin, Italy

e-mail: lorenzo.pinessi@unito.it

S. Omboni

Italian Institute of Telemedicine, Varese, Italy

C. Lisotto

Ospedale Civile, San Vito al Tagliamento, Italy

G. Zanchin

Department of Neurology, University of Padova, Padua, Italy

M. D. Ferrari

Leiden Centre for Translational Neuroscience,

Department of Neurology, Leiden University Medical Centre,

Leiden, The Netherlands

D. Zava

Istituto Lusofarmaco d'Italia, Milan, Italy
Secondary endpoints were pain-free and pain relief episodes at $2 \mathrm{~h}$, and recurrent and sustained pain-free episodes within 48 h. 104 of the 125 patients (83\%, intentionto-treat population) expressed a preference for a triptan. The average preference score was not significantly different between frovatriptan $(2.9 \pm 1.3)$ and rizatriptan $(3.2 \pm 1.1)$. The rates of pain-free $(33 \%$ frovatriptan vs. $39 \%$ rizatriptan) and pain relief (55 vs. $62 \%$ ) episodes at $2 \mathrm{~h}$ were not significantly different between the two treatments. The rate of recurrent episodes was significantly $(p<0.001)$ lower under frovatriptan $(21$ vs. $43 \%$ rizatriptan). No significant differences were observed in sustained pain-free episodes (26\% frovatriptan vs. $22 \%$ rizatriptan). The number of patients with adverse events was not significantly different between rizatriptan (34) and frovatriptan $(25, p=\mathrm{NS})$. The results suggest that frovatriptan has a similar efficacy to rizatriptan, but a more prolonged duration of action.

Keywords Migraine - Frovatriptan - Rizatriptan · Patient preference

\section{Introduction}

Migraine is a common, chronic, neurovascular disorder, typically characterized by recurrent disabling attacks of severe headache, autonomic nervous system dysfunction, and in up to a third of patients, neurological aura symptoms [1]. The triptans, selective serotonin 5-HT1B/1D agonists, are very effective acute migraine drugs [2]. The early success of sumatriptan, the parent drug of this class [3], has prompted the development of other triptan compounds, trying to optimize efficacy and safety in treatment of migraine. 
Frovatriptan is an antimigraine agent of the triptan class, developed in order to provide a triptan with a clinical potential for a long duration of action and a low likelihood of side effects and drug interactions [4-6]. Randomized placebo-controlled clinical studies and post-marketing surveys showed that most patients with migraine reported significantly improved effectiveness and tolerability ratings with frovatriptan versus placebo or previous acute therapies, including triptans, analgesics, and non-steroidal antiinflammatory drugs [7, 8].

It is widely agreed that well-designed, double-blind, randomized controlled trials that directly compare two or more triptans provide the best clinical evidence for the use of these drugs. Such trials are useful if they compare several well-standardized effectiveness measures, if they cover all available agents and doses, and if they assess adverse events, enabling the reader to judge the "trade-offs" between the compared drugs [9]. However, with the only exception of a head-to-head randomized trial versus sumatriptan [10], frovatriptan efficacy had never been tested in comparative trials with other triptans. For this reason a study was setup to compare efficacy and safety of frovatriptan versus rizatriptan, the triptan known to provide consistent relief of migraine attacks and usually preferred over other treatments because of its speed of relief $[11,12]$.

The study had been designed to assess efficacy by considering patient's preference to treatment [13, 14] and also by analyzing traditional migraine treatment endpoints.

\section{Methods}

\section{Study population}

The study included subjects of male or female gender, 18-65 years old, with a current history of migraine with or without aura, according to International Headache Society (IHS) 2004 criteria, and with at least one, but no more than six migraine attacks per month for 6 months prior to entering the study [15].

Patients could not be enrolled in the study in case of: (a) uncontrolled hypertension; (b) ischemic heart disease; (c) cardiac arrhythmias or symptomatic Wolff-ParkinsonWhite syndrome; (d) previous stroke or transient ischemic attack; (e) severe liver or renal impairment; (f) any other severe or disabling medical condition; ( $\mathrm{g}$ ) history of alcohol or analgesic or psychotropic drug abuse; (h) known hypersensitivity to study drugs; (i) previously demonstrated inadequate response to at least two triptans; (j) current use of propranolol or ergothamine (and its derivatives) as a prophylactic agent; $(\mathrm{k})$ current use or use in the previous 2 weeks of MAO-inhibitors; (1) use of either test medication to treat any one of the last three episodes of migraine; and (m) other headaches that have been lasting for more than 6 days. Pregnant women and breast-feeding mothers were excluded as well, while women with childbearing potential but not practicing an effective method of birth control were to be submitted to a pregnancy test, if clinically indicated.

Written informed consent was obtained from all patients prior to their inclusion into the study. The study was approved by the Independent Institutional Review Boards of the study centers.

Study design

This was a multicenter, randomized, double-blind, crossover study, including 15 centers across Italy (Appendix). Each patient received frovatriptan $2.5 \mathrm{mg}$ or rizatriptan $10 \mathrm{mg}$ in a balanced computer-generated randomized sequence $(1: 1)$, where frovatriptan had to be followed by rizatriptan or viceversa. After treating three episodes of migraine in no more than 3 months with the first treatment, the patient had to switch to the other treatment. Randomization was done by blocks of 4 . Blindness was ensured by the overencapsulation technique, i.e., by inserting study drug tablets in capsules. After treating three episodes of migraine in no more than 3 months with the second treatment, each patient was asked to assign preference to one of the treatments according to a questionnaire with a preference score graded from 0 to 5 .

The study involved three visits and each patient's participation time in the study had not to exceed 6 months from randomization. Subjects having no migraine episodes during one of the two observation periods were excluded from the study.

During the randomization visit, after signing written informed consent, subjects provided medical, medication, and migraine history. Physical and neurological examinations and pregnancy test (if appropriate) were performed. Blood pressure and heart rate were measured for all subjects. The degree of migraine-associated disability (MIDAS questionnaire) was also completed. At the end of the visit a headache diary documenting characteristics of headache pain and associated symptoms was dispensed with study medication. Subjects were instructed to treat at least three migraine episodes occurring in no more than 3 months and to come for the second visit. On this occasion use of concomitant medications and occurrence of adverse events (from diary) were checked, blood pressure and heart rate were recorded, and a pregnancy test performed, if deemed necessary. The same procedures were carried out at the end of the second study treatment period or at the early withdrawal visit, together with the administration of the patient's preference questionnaire.

Patients were instructed to take one dose of study medication as early as possible after the onset of migraine attack. 
If insufficient relief had been obtained after $2 \mathrm{~h}$, patients were allowed to take a second dose of study medication, with a maximum daily intake of two doses. In case of insufficient relief $1 \mathrm{~h}$ after the intake of the second dose of the study medication, patients were allowed to take a rescue medication. Alternate rescue medication could not include triptans, or contain ergotamine or its derivatives, or propranolol.

\section{Data analysis}

The primary study endpoint was the between-treatment comparison of the direction and average strength of preference at the end of the study, measured on a scale from 0 (no preference) to 5 (strong preference) [14]. The rate of patients expressing a preference and reason for preference were also calculated. The preference scale and questionnaire are reported in Fig. 1 and Appendix 1 (Supplementary material).

The hypothesis was that a superiority of one treatment against the other had to occur in the presence of a difference of +1.0 with a standard deviation of 2.375 . Considering a two-tailed test with a 0.05 significance level and an $80.7 \%$ power, the estimated number of patients to be randomized was 120 (including a 25\% drop-outs), 60 for each treatment group.

The intention-to-treat population, including all patients who treated at least one attack in each treatment period and completed the preference questionnaire, was the study primary analysis population. The per-protocol population consisted of all patients treating the three anticipated migraine episodes in a maximum of 3 months during both treatment periods and who expressed their preference at study termination, with no protocol violations. This population was used for confirmatory analysis.

Secondary study endpoints were quantified according to IHS Guidelines [15]. In summary, these endpoints were (a) the number of pain-free episodes at $2 \mathrm{~h}$ (absence of migraine episodes at $2 \mathrm{~h}$ after intake of one dose of study drug), (b) recurrence, assessed as stated in the protocol, i.e., an episode of migraine occurring within $48 \mathrm{~h}$ from the

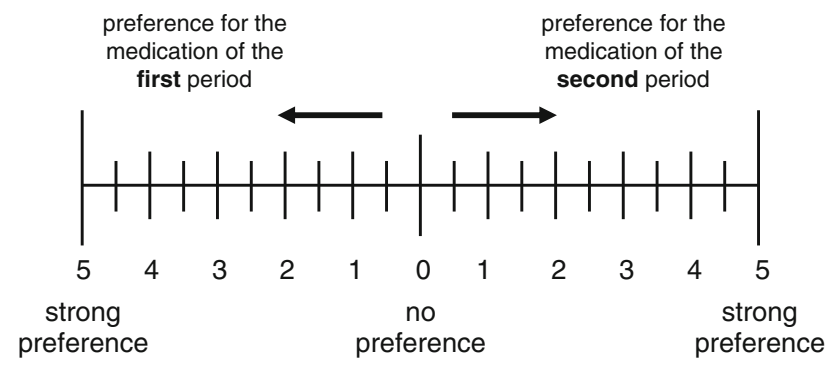

Fig. 1 Example of preference scoring. For details on the contents of the preference questionnaire, please refer to Appendix 1 (Supplementary material) previous one, after a period without migraine, and also more appropriately as an episode which is pain free at $2 \mathrm{~h}$ and headache of any severity returns within $48 \mathrm{~h}$, without taking a second dose or a rescue medication; (c) the number of sustained pain-free episodes within $48 \mathrm{~h}$ (migraine attack which is pain free at $2 \mathrm{~h}$, does not recur and does not require the use of rescue medication within $48 \mathrm{~h}$ ), (d) the number of pain relief episodes at $2 \mathrm{~h}$ (defined as a decrease in migraine intensity from severe or moderate to mild or none). Consistency of response (responders in 2 and 3 out of 3 attacks) was assessed for the secondary endpoints. An evaluation of the total amount of working hours lost due to migraine attacks and the rate of occurrence of nausea, vomiting, photophobia, phonophobia, and osmophobia 2, 4, 24, and $48 \mathrm{~h}$ after drug intake, were also assessed.

Safety analysis was applied to all randomized patients, by calculating the incidence of adverse events and changes in vital signs during the study.

Continuous variables were summarized by computing average values and standard deviation (SD), while categorical variables by computing the absolute value and the frequency (as percentage). Preference scores were compared between-treatment groups by analysis of variance. Secondary endpoints were compared between groups by generalized estimating equation analysis. Kaplan-Meyer curves for cumulative hazard of recurrence over $48 \mathrm{~h}$ were also drawn. $p$ value refers to the statistical significance of between-treatment difference. The level of statistical significance was kept at 0.05 throughout the whole study.

\section{Results}

Baseline demographic and clinical data

Overall, 148 patients were screened and randomized to active treatment. Of these patients, 129 completed the study and 19 prematurely withdrew from the study because of dissatisfaction to assigned treatment $(n=1)$, withdrawal of consent $(n=6)$, failure to treat one episode of migraine $(n=6)$, occurrence of an adverse event $(n=2)$, protocol violation $(n=1)$, deterioration of target disease symptoms $(n=1)$, or other reasons $(n=2)$. A flow diagram of the patients throughout the study is reported in Fig. 2.

The intention-to-treat population consisted of 125 patients, while patients valid for per-protocol analysis were 96. Safety analysis was carried out in 137 patients.

Table 1 shows main demographic and clinical characteristics of patients of the intention-to-treat. Most of the subjects enrolled were females. No patients with migraine with aura reported previous treatment with triptans. Similar results were observed for the per-protocol population. 


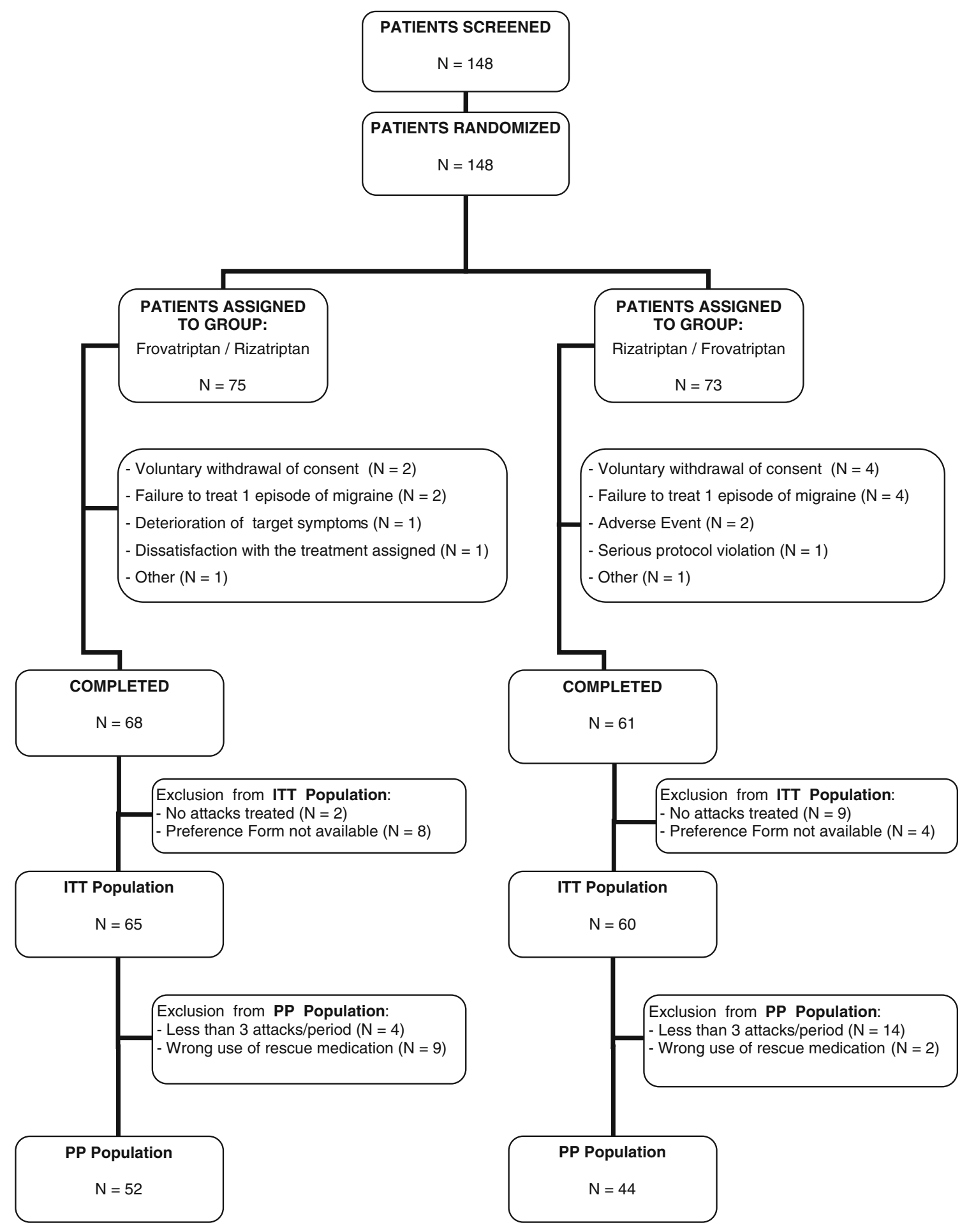

Fig. 2 Flow diagram of participants throughout the study

Primary end point

In the intention-to-treat population, the preference score averaged to $2.9 \pm 1.3$ for frovatriptan and to $3.2 \pm 1.1$ for rizatriptan ( $p=\mathrm{NS})$.
A total of $104(83 \%)$ patients expressed a clear preference for a triptan: 42 (38\%) for frovatriptan and 40 (45\%) for rizatriptan ( $p=$ NS between treatments).

The reasons for preferring one triptan in the intentionto-treat population were the rapid action (71\% frovatriptan 
Table 1 Demographic and clinical data of the 125 patients of the intention-to-treat population and of the 96 patients of the per-protocol population at the time of randomization

\begin{tabular}{lll}
\hline & $\begin{array}{l}\text { ITT } \\
(n=125)\end{array}$ & $\begin{array}{l}\text { PP } \\
(n=96)\end{array}$ \\
\hline Age (years, mean $\pm \mathrm{SD})$ & $37 \pm 9$ & $37 \pm 9$ \\
Females $(n, \%)$ & $99(79)$ & $75(78)$ \\
Height $(\mathrm{cm}$, mean $\pm \mathrm{SD})$ & $167 \pm 9$ & $167 \pm 9$ \\
Weight $(\mathrm{kg}$, mean $\pm \mathrm{SD})$ & $64 \pm 13$ & $64 \pm 13$ \\
Age at onset of migraine (years, mean $\pm \mathrm{SD})$ & $16 \pm 7$ & $16 \pm 7$ \\
Migraine attack duration $>2$ days $(n, \%)$ & $26(21)$ & $14(15)$ \\
MIDAS score (mean $\pm \mathrm{SD})$ & $22 \pm 15$ & $21 \pm 16$ \\
Migraine with aura $(n, \%)$ & $4(3)$ & $3(3)$ \\
\hline
\end{tabular}

Data are shown as mean $( \pm \mathrm{SD})$, or absolute $(n)$ and relative frequency $(\%)$

vs. $66 \%$ rizatriptan), followed by reduction in migraine severity (33 vs. $54 \%$ ), complete analgesia (33 vs. $54 \%$ ), functional recovery (33 vs. $36 \%$ ), and improved tolerability (42 and 38\%). No significant differences were observed between treatments. Additional preference results will be published in details elsewhere. Results of analysis of the per-protocol population did not differ from those of the intention-to-treat group.

\section{Secondary endpoints}

Results of the analysis of secondary endpoints are summarized in Table 2. In the intention-to-treat population, the rates of pain-free (33\% with frovatriptan vs. $39 \%$ with rizatriptan) and pain relief episodes at $2 \mathrm{~h}(55 \%$ with frovatriptan vs. $62 \%$ with rizatriptan) were not significantly $(p=\mathrm{NS})$ different between the two treatments (Table 2). Rate of recurrent episodes was significantly lower under frovatriptan, either when defined according to the protocol (22 vs. $32 \%$ rizatriptan; $p<0.001$ ) or to IHS indications (21 vs. $43 \% ; p<0.001$ ). This was confirmed by a significantly $(p<0.05)$ lower cumulative hazard of recurrence over the $48 \mathrm{~h}$ (Fig. 3).
Sustained pain-free episodes were also similar between the two groups (26\% frovatriptan vs. $22 \%$ rizatriptan; $p=\mathrm{NS}$ ) (Table 2). No difference was observed between the intention-to-treat and the per-protocol population for main secondary endpoints (Table 2).

Consistency of response for secondary endpoints resulted statistically significant in favor of frovatriptan for pain relief at $2 \mathrm{~h}$ (Table 3 ).

Treatment with frovatriptan had also a positive impact on the amount of working hours lost due to migraine attacks, which was significantly lower with this drug $(1.5 \pm 3.1 \mathrm{~h})$ than with rizatriptan $(2.8 \pm 9.4 \mathrm{~h}, p<0.05)$ in the intention-to-treat population. Number of concomitant medications was similar between the two study sequences (130 for frovatriptan-rizatriptan sequence and 135 for rizatriptan-frovatriptan sequence). Also use of second dose and rescue medication, and severity of headache at the time of study drug intake did not significantly differ between study groups (Table 4).

Both drugs gradually reduced migraine-related symptoms, with no differences between treatments (Fig. 4).

\section{Safety}

Safety analysis was carried out in 137 patients. Adverse events were reported by 25 patients during treatment with frovatriptan and by 34 patients during treatment with rizatriptan (18.8 vs. $26.2 \%$ of treated patients,) for an overall number of 158 adverse events (65 under frovatriptan and 93 under rizatriptan). Most of the events were of a mild or moderate intensity, and no serious adverse events were recorded during the study. No patients under Frovatriptan prematurely withdrew from the study while two patients under Rizatriptan did.

Side effects attributed to study treatment were 89 (56.3\% of total events) and occurred in 13 patients during frovatriptan and in 15 patients during rizatriptan treatment $(p=\mathrm{NS})$. As reported in Table 5 the number of drugrelated adverse events was slightly, but not significantly, greater under rizatriptan than frovatriptan.

Table 2 Result for the secondary study endpoints

\begin{tabular}{|c|c|c|c|c|c|c|}
\hline & \multicolumn{2}{|c|}{ ITT $(n=125)$} & \multirow[t]{2}{*}{$p$} & \multicolumn{2}{|l|}{$\mathrm{PP}(n=96)$} & \multirow[t]{2}{*}{$p$} \\
\hline & Frovatriptan & Rizatriptan & & Frovatriptan & Rizatriptan & \\
\hline Pain-free episodes at $2 \mathrm{~h}$ & $117(33)$ & $141(39)$ & NS & 107 (37) & $116(40)$ & NS \\
\hline Recurrent episodes (IHS) & $25(21)$ & $61(43)$ & $<0.001$ & $21(20)$ & $40(35)$ & $<0.05$ \\
\hline Recurrent episodes (protocol) & $77(22)$ & $117(32)$ & $<0.001$ & $61(21)$ & $91(32)$ & $<0.01$ \\
\hline Sustained pain-free episodes & $92(26)$ & $80(22)$ & NS & $86(30)$ & $76(26)$ & NS \\
\hline Pain relief episodes at $2 \mathrm{~h}$ & $155(55)$ & $166(62)$ & NS & $129(61)$ & $134(60)$ & NS \\
\hline
\end{tabular}

Data are shown for the intention-to-treat and per-protocol population and reported as absolute $(n)$ and relative $(\%)$ frequency. $p$ refer to the statistical significance of the difference between the two treatment groups 


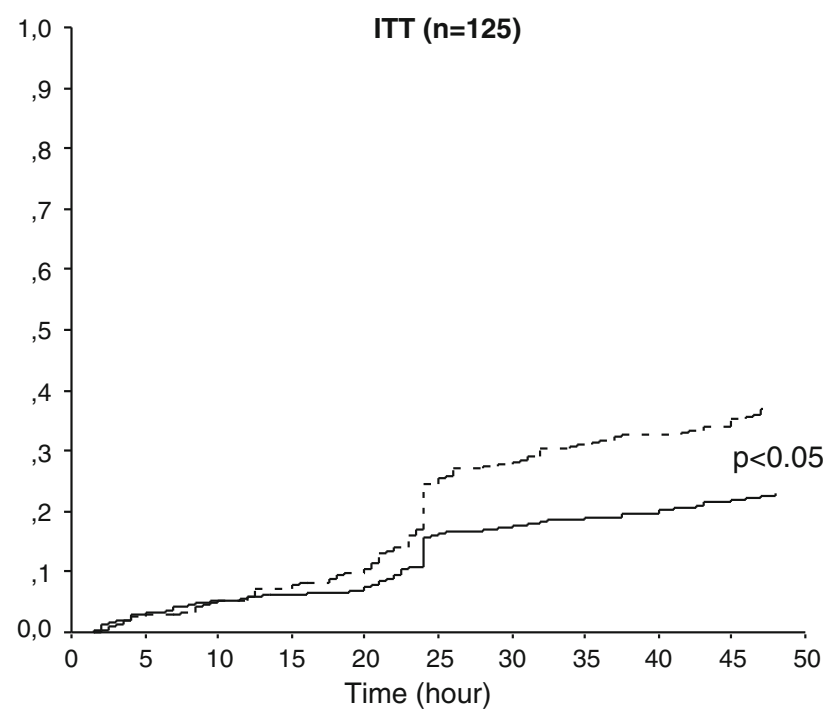

Fig. 3 Cumulative hazard of recurrence over the $48 \mathrm{~h}$ during treatment with frovatriptan (continuous line) or rizatriptan (dashed line), in the 125 patients of intention-to-treat population. Recurrence was defined according to protocol

Table 3 Result of consistency analysis for the secondary study endpoints

\begin{tabular}{lcrl}
\hline & \multicolumn{2}{l}{ ITT $(n=125)$} & \multirow{2}{*}{$p$} \\
\cline { 2 - 3 } & Frovatriptan & Rizatriptan & \\
\hline Pain-free episodes at 2 h & $88(75)$ & $108(77)$ & NS \\
Recurrent episodes (IHS) & $13(52)$ & $31(51)$ & NS \\
Recurrent episodes (protocol) & $49(64)$ & $85(73)$ & NS \\
Sustained pain-free episodes & $61(66)$ & $57(71)$ & NS \\
Pain relief episodes at 2 h & $132(85)$ & $127(77)$ & $<0.05$ \\
\hline
\end{tabular}

Consistency of response was defined as responders in 2 and 3 out of 3 attacks. Data are shown for the intention-to-treat population and reported as absolute $(n)$ and relative (\%) frequency. $p$ refer to the statistical significance of the difference between the two treatment groups

Table 4 Migraine attacks with use of two or more doses, rescue medication, and severity of headache at the time of drug intake

\begin{tabular}{llll}
\hline & \multicolumn{2}{l}{ ITT $(n=125)$} & $p$ \\
\cline { 2 - 3 } & $\begin{array}{l}\text { Frovatriptan } \\
(n=357)\end{array}$ & $\begin{array}{l}\text { Rizatriptan } \\
(n=362)\end{array}$ & \\
\hline Use of 2 doses of study medication & $110(31)$ & $122(34)$ & NS \\
Use of $>2$ doses of study medication & $67(19)$ & $74(20)$ & NS \\
Use of rescue medication & $71(20)$ & $59(16)$ & NS \\
Severity of headache at study drug intake & & \\
Mild & $76(21)$ & $93(25)$ & NS \\
Moderate & $203(57)$ & $201(56)$ & NS \\
Severe & $78(22)$ & $68(19)$ & NS \\
\hline
\end{tabular}

Data are shown for the intention-to-treat population and reported as absolute (n) and relative (\%) frequency. $p$ refer to the statistical significance of the difference between the two treatment groups
Treatment was accompanied by no relevant changes in vital signs, ECG, or results of cardiovascular examination.

\section{Discussion}

In this direct comparative study of frovatriptan versus rizatriptan in acute migraine, the two drugs showed a similar efficacy, but frovatriptan had a more sustained analgesic effect. Our results were consistent for both the intention-to-treat and per-protocol population.

Patient's preference for one drug or the other did not differ between the study treatments. Frovatriptan was chosen mainly because of the rapid speed of action ( $71 \%$ of patients), good tolerability ( $42 \%$ of patients), and reduction in pain severity (33\%). The fact that approximately $70 \%$ of patients preferred frovatriptan for its rapid activity confirms what was recently reported in a long-term open label study, namely that frovatriptan may provide a remarkably fast and high headache response in more than one-third of migraineurs, labeled as "rapid responders" [16].

A relevant result of our study is that recurrence rate within $48 \mathrm{~h}$ were significantly lower under frovatriptan than under rizatriptan. These differences may be explained by the different pharmacokinetics of the two drugs. Indeed, earlier studies have shown that, due to its shorter time to maximum concentration, rizatriptan tends to produce a quicker onset of headache relief and a faster freedom from pain than sumatriptan (the reference drug of the class), zolmitriptan, and naratriptan [6, 17]. For this reason rizatriptan is now widely regarded by many physicians as a first-line treatment option for the management of migraine [17]. Conversely, frovatriptan has a time to maximum concentration typically of 2 to $3 \mathrm{~h}$, but the longest half-life among triptans, greater $5-\mathrm{HT}_{1 \mathrm{~B}}$ binding receptor potency, and multiple pathways metabolism. These pharmacological features might explain differences in clinical efficacy between the two drugs and why frovatriptan, unlike other triptans, including rizatriptan, greatly reduced the risk of migraine recurrence [18-21].

Though previous direct comparisons between frovatriptan and rizatriptan are not available, our results are in line with those of previous placebo-controlled studies based on these two triptans [22, 23]. As far as frovatriptan is regarded, in a review of five randomized, double-blind, placebo-controlled studies, headache response rate at $2 \mathrm{~h}$ (pain relief) for frovatriptan ranged between 38 and 40\% before placebo correction, while it was slightly higher in our study (55\%) [22]. In previous studies frovatriptan was more effective than placebo in rendering patient pain free at 2 (12\% of patients) and $4 \mathrm{~h}$ (29\% of patients) [4, 7]. The additional finding of our study is that proportion of painfree episodes at $2 \mathrm{~h}$ was much higher than that observed in 
Fig. 4 Frequency $(\%)$ of occurrence of nausea, vomiting, photophobia, phonophobia, and osmophobia 2, 4, 24, and $48 \mathrm{~h}$ after administration of frovatriptan (open bars) or rizatriptan (striped bars) in the 125 patients of the intention-totreat population

Table 5 Distribution of absolute numbers of drugrelated adverse events between the two treatment groups in the 137 patients of the safety analysis

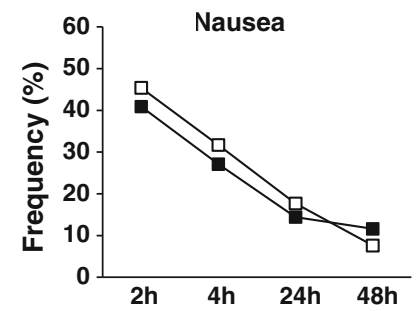

ITT ( $n=125)$
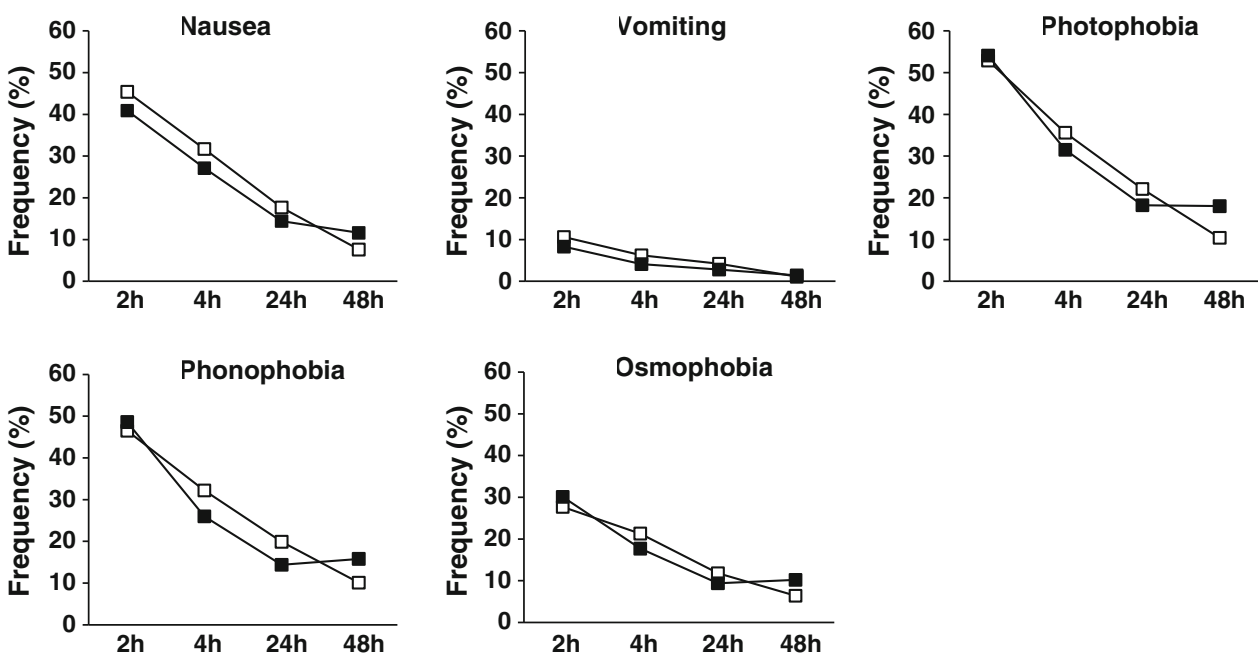

\begin{tabular}{|c|c|c|c|c|c|c|c|}
\hline & \multicolumn{6}{|c|}{ Intensity } & \multirow{3}{*}{$\begin{array}{l}\text { All } \\
(n=137)\end{array}$} \\
\hline & \multicolumn{3}{|c|}{ Frovatriptan $(n=133)$} & \multicolumn{3}{|c|}{ Rizatriptan $(n=130)$} & \\
\hline & Mild & Moderate & Severe & Mild & Moderate & Severe & \\
\hline Asthenia & 3 & 5 & 2 & 1 & 4 & 1 & 16 \\
\hline Somnolence & 3 & - & - & - & 6 & 5 & 14 \\
\hline Dizziness & 1 & - & - & 2 & 4 & 1 & 8 \\
\hline Nausea or vomiting & - & - & 3 & - & 1 & - & 4 \\
\hline Throat tightness & 1 & - & - & 2 & 2 & 3 & 8 \\
\hline Pain increase & - & 1 & 3 & - & - & 2 & 6 \\
\hline Burning sensation to nose and forehead & 1 & 1 & 3 & - & 1 & - & 6 \\
\hline Palpitation or tachycardia & - & - & - & 2 & 2 & - & 4 \\
\hline Sensation of being dazed & 2 & 1 & - & - & 1 & - & 4 \\
\hline Instability or imbalance & - & - & - & 1 & 3 & - & 4 \\
\hline Abdominal pain & - & - & 1 & - & - & - & 1 \\
\hline Muscular or bone pain & - & 2 & - & - & - & - & 2 \\
\hline Thoracic constriction & - & - & - & - & 1 & - & 1 \\
\hline Tremor or shivers & - & - & - & 1 & 1 & - & 2 \\
\hline Other & 1 & 2 & 3 & - & 2 & 1 & 9 \\
\hline Total adverse events & & 39 & & & 50 & & 89 \\
\hline Total patients (\%) & & $13(9.8)$ & & & $15(11.5)$ & & $28(20.4)$ \\
\hline
\end{tabular}

previous placebo-controlled studies (33 vs. 9-14\%). Headache recurrence at $24 \mathrm{~h}$ averaged to $17 \%$ in a previous meta-analysis [20]: in our study headache recurrence was assessed more properly within the $48 \mathrm{~h}$, as indicated by IHS guidelines [15], and was significantly less frequent than under rizatriptan. This is in line with results of open label naturalistic studies and meta-analyses of placebocontrolled efficacy studies, which suggest that in a realworld setting frovatriptan is associated with a lower rate of migraine recurrence than with other triptans [24]. Postmarketing surveys also indicate that the long duration of action of frovatriptan appears to confer other benefits such as greater patient satisfaction, with over $85-90 \%$ of patients and physicians rating frovatriptan therapy as very good or good, both in terms of efficacy and tolerability [8, 24].

The lower recurrence of headache after $48 \mathrm{~h}$ from drug intake with frovatriptan seems to support indication of frovatriptan for those patients requiring a prolonged duration of action, with a sustained effect. Pharmacokinetic features of frovatriptan may make it suitable for patients who need prophylaxis such as in menstrual-related migraine 
and for patients typically suffering from migraines of long duration or with frequent recurrence $[4,25,26]$. In these patients, early use of frovatriptan has been shown to be associated with a greater benefit [27]. Conversely, rizatriptan may be useful for those patients requiring a rapid pain relief. The prolonged analgesic effect of frovatriptan is also reflected in a smaller number of working hours lost due to migraine under treatment with these drugs.

In conclusion, our multicenter, randomized, doubleblind study suggests that frovatriptan and rizatriptan are similarly preferred in migraineurs patients and share a similar antimigraine efficacy, though the former seems to have a more prolonged and sustained analgesic effect.

Acknowledgments The present study was supported by Istituto Lusofarmaco d'Italia.

Conflict of interest All authors have occasionally served as scientific consultants for manufacturers of frovatriptan or rizatriptan. Dario Zava is an employee of the manufacturer of frovatriptan.

\section{Appendix}

List of study sites

\section{Coordinator}

Prof. L. Pinessi (Torino)

Investigators

P. De Martino (Torino), B. Panascia (Palermo), R. Rapisarda (Palermo), F. Devetag (Feltre), M.G. Sances (Pavia), L.A. Pini (Modena), G. Bono (Varese), R. Cerbo (Roma), M. De Marinis (Roma), M. Guidotti, R. Ravasio (Como), M. Alessandri (Grosseto), E. De Caro (Catanzaro), F. Lanaia (Catania), M.P. Prudenzano (Bari), G. Reggiardo (Biostatistical Unit, Mediservice, Milano), F. Sacchi (Clinical Unit, Mediservice, Milano)

\section{References}

1. Ferrari MD (1998) Migraine. Lancet 351:1043-1051

2. Rapoport AM (2006) Acute treatment of headache. J Headache Pain 7:339-355

3. Cady R, Schreiber C (2006) Sumatriptan: update and review. Expert Opin Pharmacother 7:1503-1514

4. Balbisi EA (2004) Frovatriptan succinate, a 5-HT1B/1D receptor agonist for migraine. Int J Clin Pract 58:695-705

5. Buchan P, Wade A, Ward C, Oliver SD, Stewart AJ, Freestone S (2002) Frovatriptan: a review of drug-drug interactions. Headache 42(Suppl.2):S63-S73
6. Tfelt-Hansen P, De Vries P, Saxena PR (2000) Triptans in migraine: a comparative review of pharmacology, pharmacokinetics and efficacy. Drugs 60:1259-1287

7. Poolsup N, Leelasangaluk V, Jittangtrong J, Rithlamlert C, Ratanapantamanee N, Khanthong M (2005) Efficacy and tolerability of frovatriptan in acute migraine treatment: systematic review of randomized controlled trials. J Clin Pharm Ther 30:521-532

8. Cady RK, Banks J, Jones BA, Campbell J (2009) Postmarketing migraine survey of frovatriptan: effectiveness and tolerability vs previous triptans, NSAIDs or a combination. Curr Med Res Opin 25:2711-2721

9. Goadsby PJ (1999) The scientific basis of medication choice in symptomatic migraine treatment. Can J Neurol Sci 26(Suppl.3): S20-S26

10. Géraud G, Spierings EL, Keywood C (2002) Tolerability and safety of frovatriptan with short- and long-term use for treatment of migraine and in comparison with sumatriptan. Headache 42(Suppl.2):S93-S99

11. Hargreaves RJ, Lines CR, Rapoport AM, Ho TW, Sheftell FD (2009) Ten years of rizatriptan: from development to clinical science and future directions. Headache 49(Suppl.1):S3-S20

12. Mannix LK (2008) A review of the 5-HT1B/1D agonist rizatriptan: update on recent research and implications for the future. Expert Opin Pharmacother 9:1001-1011

13. Dahlöf C (2001) Assessing patient preference in migraine treatment. Cephalalgia 21:791-795

14. Tullo V, Allais G, Ferrari MD, Curone M, Mea E, Omboni S, Benedetto C, Zava D, Bussone G (2010) Frovatriptan versus zolmitriptan for the acute treatment of migraine: a double-blind, randomized, multicenter, Italian study. Neurol Sci 31(Suppl.1): S51-S54

15. Headache Classification Subcommittee of the International Headache Society (2004) The International Classification of Headache Disorders, 2nd edn. Cephalalgia 24(Suppl.1):9-160

16. Spierings EL, Keywood C (2009) Rapid responders to frovatriptan in acute migraine treatment: results from a long-term, open-label study. Pain Med 10:633-638

17. Wellington K, Plosker GL (2002) Rizatriptan: an update of its use in the management of migraine. Drugs 62:1539-1574

18. Markus F, Mikko K (2007) Frovatriptan review. Expert Opin Pharmacother 8:3029-3033

19. Dooley M, Faulds D (1999) Rizatriptan: a review of its efficacy in the management of migraine. Drugs 58:699-723

20. Géraud G, Keywood C, Senard JM (2003) Migraine headache recurrence: relationship to clinical, pharmacological, and pharmacokinetic properties of triptans. Headache 43:376-388

21. Buchan P, Keywood C, Wade A, Ward C (2002) Clinical pharmacokinetics of frovatriptan. Headache 42(Suppl 2):S54-S62

22. Ferrari MD, Goadsby PJ, Roon KI, Lipton RB (2002) Triptans (serotonin, 5-HT1B/1D agonists) in migraine: detailed results and methods of a meta-analysis of 53 trials. Cephalalgia 22:633-658

23. Adelman JU, Belsey J (2003) Meta-analysis of oral triptan therapy for migraine: number needed to treat and relative cost to achieve relief within 2 hours. J Manage Care Pharm 9:45-52

24. Guidotti M, Ravasio R (2009) Clinical and economic comparison of frovatriptan versus other oral triptans in the treatment of acute migraine in the real-world setting. Clin Drug Investig 29:693-702

25. Kelman L (2008) Review of frovatriptan in the treatment of migraine. Neuropsychiatr Dis Treat 4:49-54

26. Balbisi EA (2006) Frovatriptan: a review of pharmacology, pharmacokinetics and clinical potential in the treatment of menstrual migraine. Ther Clin Risk Manag 2:303-308

27. Cady R, Elkind A, Goldstein J, Keywood C (2004) Randomized, placebo-controlled comparison of early use of frovatriptan in a migraine attack versus dosing after the headache has become moderate or severe. Curr Med Res Opin 20:1465-1472 